\title{
Rejection of Manuscripts
}

\section{K.C. S}

High quality scientific work is the key to publication in any journal. The most important thing is to start writing for the journal. Writing scientific manuscripts can be unnecessarily daunting, if not paralyzing. This paralysis is usually the result of one of two reasons: either researchers do not know how to start, or they do not know what to put where. ${ }^{1}$ Writing a research paper is essentially methodic. Guidelines for structure and organization can be followed to make the process much more straightforward. The essentials of any paper include a description of what is known, an assessment of what is unknown, a clear statement regarding the question and hypothesis being addressed by the current study, and a discussion and summary of new information that has been learned as a result of the study. ${ }^{2}$ Failure to do so or not following the instructions to the author properly can lead to rejection of the manuscript. However, it is rare to find an author who has not, at some point, received a notice from a journal that a manuscript must be substantially revised before it can be published or one that states that the manuscript is rejected. ${ }^{3}$ Not to despair, you may reassure yourself from the real fact that even the eminent writer's papers get rejected or require major revisions before they are published. Nobody can claim to be perfect in manuscript writing. Designing an appropriate study that answers a clearly defined and pertinent question is an important first step for acceptance of a manuscript. ${ }^{4}$ It is rewarding for the author (who learns the most), and the intended reader, who will use the information and the science to move the field of medicine along and to optimize medical care. ${ }^{5}$

Acceptance or rejection rates vary from journal to journal, and are dependent on a variety of factors. Rejection from the Editorial board of Journal of Medical Association of Nepal (JNMA) before sending to the reviewer is on increase these days. Need of publication in indexed journal for various reasons are on rise, leading to increase in numbers of submission in recent years. There are many reasons for rejection of manuscripts in JNMA. Failure to follow the JNMA guideline is the most common factor. Old findings described elsewhere but presented as new finding in particular institution with no new or useful material is another reason. Fundamentally weak hypothesis with lack of clinical relevance is one more reason for us to reject the manuscript. Sample population too small or biased to justify results and conclusion are sometimes found to be the reason. We also sometimes come across with too poorly written, phrased, or presented manuscripts, principally because English is not the primary language of the author. Many reviewers believe that the language barrier is an important factor in manuscript rejection. Nevertheless, the language barrier is not the sole factor. We, at JNMA, do not consider language problem as the prime cause of rejection. We feel that language problems are correctable and should not be the major reason for rejection. We also have encountered plagiarism, mostly in review article, as primary reason for rejection. Last but not the least poor statistics and poor application of statistical tools are sometimes encountered.

The peer review system is an important component of modern scientific publishing while the reviewers are key player in the journal publication process. Peer reviewers aim to provide a critical, independent and unbiased assessment of submitted manuscripts, and are regarded as an important extension of the scientific process. ${ }^{6}$ In this issue of JNMA, we have include an article by a regular reviewer entitled "What do reviewers look for in an original research article?". ${ }^{7}$ This article will be a great help to the authors,

Correspondence:

Dr. Sudhamshu K.C.

Liver unit Department of Medicine

NAMS, Bir Hospital

Kathmandu, Nepal

Email:sudhamshu.liver@gmail.com 
specially the new ones. Following instructions to author (JNMA) properly and keeping in mind the points discussed by the reviewer will definitely decrease the chances of rejection.

The manuscript can always be improved by incorporating changes as suggested by the editors and reviewers. The comments and the suggestions given by the reviewer should be taken as gift of knowledge, not as humiliation. The knowledge gained in the review process should be used logically and it makes subsequent acceptance of your article in another journal much easier.

\section{REFERENCES}

1. Kliewer MA.Writing it up: a step-by-step guide to publication for beginning investigators.AJR Am J Roentgenol. 2005;185:591-6.

2. Cetin S, Hackam DJ. An approach to the writing of a scientific manuscript.J Surg Res. 2005 Oct;128,2:165-7.

3. Provenzale JM. Revising a manuscript: ten principles to guide success for publication. Am J Roentgenol. 2010;195,6:W382-7.

4. Ehara S, Takahashi K. Reasons for rejection of manuscripts submitted to AJR by international authors.AJR Am J Roentgenol. 2007;188,2:W113-6.

5. Holmes DR Jr, Hodgson PK, Nishimura RA, Simari RD. Manuscript preparation and publication. Circulation. 2009;120,10:906-13

6. International Committee of Medical Journal Editors. Uniform requirements for manuscripts submitted to biomedical journals. Updated October 2008. Available at: www.icjme.org.

7. Shanker PR. What do reviewers look for in an original research article? J Nepal Med Assoc 2012; 52(186) 95-101 